\title{
DISTRIBUCIÓN ESPACIAL Y PERMANENCIA TEMPORAL DE HORMIGUEROS EN EL AGROSISTEMA DEL OLIVO EN GRANADA, ESPAÑA
}

\section{ANT NESTS SPATIAL DISTRIBUTION AND TEMPORARY PERMANENCE IN OLIVE ORCHARDS AT GRANADA, SPAIN.}

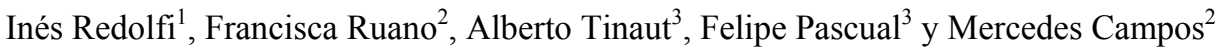

\begin{abstract}
Resumen
El estudio se realizó en tres olivares con diferente manejo agronómico, a $20 \mathrm{~km}$ de la ciudad de Granada. En cada olivar se observó el área correspondiente a 60 árboles (seis hileras contiguas de 10 árboles) y cinco transectos de $100 \mathrm{~m}$ de longitud por un metro de ancho en las calles, entre las hileras de árboles. En el suelo bajo la copa de cada árbol y en las calles se determinó la ubicación de los hormigueros, en un área total de $540 \mathrm{~m}^{2}\left(9 \mathrm{~m}^{2} /\right.$ árbol $)$ y $500 \mathrm{~m}^{2}\left(100 \mathrm{~m}^{2} / \mathrm{calle}\right)$ respectivamente. Se procedió al mapeo de los nidos durante los meses de Mayo, Julio y Septiembre de 1997 y Mayo de 1998 y a medir la distancia entre nidos en los tres meses de 1997. La determinación de la distribución de los hormigueros se realizó por medio del método del vecino más próximo. Los resultados indican que la distribución de los nidos de las principales especies (Aphaenogaster senilis, Messor barbarus, Crematogaster auberti, Crematogaster sordidula, Solenopsis latro, Tetramorium semilaeve, Tapinoma nigerrima, Plagiolepis pygmaea, Cataglyphis rosenhaueri) y del total de los nidos en el olivar, corresponde en general a una distribución regular que pasa al azar cuando la densidad disminuye. Los Indices de Permanencia intraanual de los hormigueros fueron bajos $(0.0$ a $57.1 \%$ ) y los interanual con valor cero. Los Indices de Renovación de los nidos variaron entre $0 \%$ y $100 \%$.

Palabras claves: Formicidae, distribución espacial, permanencia temporal de nidos, olivar, diferente manejo agronómico
\end{abstract}

\begin{abstract}
Ant nests were studied in three olive orchards under different agricultural managements located $20 \mathrm{~km}$ from Granada City. In each orchard, the area covered by 60 trees -six 10 trees contiguous rows- and five $100 \mathrm{~m}$ long and $1 \mathrm{~m}$ wide transects in the alleyways among the rows were monitored. In the ground under each tree crown and in the alleyways, the position of the nests were determined covering an area of $540 \mathrm{~m}^{2}\left(9 \mathrm{~m}^{2} /\right.$ tree $)$ and of $500 \mathrm{~m}^{2}\left(100 \mathrm{~m}^{2} /\right.$ alley) respectively. Nest positions were assessed during May, July and September of 1997 and May of 1998. The distance between ant nests was mapped in the three 1997 months. Ant nests distribution was obtained with the distance to the nearest neighbour method. Results show regular nests distributions for the main ant species -Aphaenogaster senilis, Messor barbarus, Crematogaster auberti, Crematogaster sordidula, Solenopsis latro, Tetramorium semilaeve, Tapinoma nigerrima, Plagiolepis pygmaea, Cataglyphis rosenhaueri- and for all of the species in the orchards but it may turn into a random distribution when the nest density is decreased. The intraannual nest permanence indeces were low $(0.0 \%$ to $57 \%)$ and the inter-annual nests permanence indeces were $0 \%$. The nest renovation indeces ranged from $0.0 \%$ to $100 \%$.

Keys words: Formicidae, nests spacial distribution, temporary permanence, olive orchards, different agricultural management.
\end{abstract}

\section{Introducción}

Las hormigas ocupan un importante lugar en los ecosistemas porque se estima que el flujo de energía en sus poblaciones excede la de los vertebrados homeotermos que conviven en el mismo habitat (Petal, 1978), y el amplio rango de dimensiones en los nichos les permiten intervenir en el ciclo de nutrientes en la naturaleza, en el enriquecimiento de los suelos, en una gran diversidad de interacciones tróficas y funcionar como agentes en el control biológico natural de plagas, como bioindicadores y como elementos para indicar la biodiversidad integral (Adlung, 1966; Majer, 1972, 1983, 1994; El Haidari, 1981; Andersen, 1990; Detrain, 1990; Burbidge et al., 1992; Paulson \& Akre, 1992; Way \& Khoo, 1992; Weseloh, 1993, 1996; Tinaut et al., 1994; Majer \& Beeston, 1996; Peng et al., 1997; Rabitsch, 1997; Morris, 1997; Redolfi, 1999; Morris et al., 1998 a; Morris et al., 2002). El estudio de las hormigas se desarrolló en ecosistemas naturales, incluyendo posteriormente los agrosistemas de árboles (Majer, 1972; Majer \& Delabie, 1993; Fiala et al., 1994; Majer \& Delabie, 
1994; Perfecto \& Vandermeer, 1994; Perfecto \& Snelling, 1995). La importancia de las hormigas en el cultivo de olivo (Oleae europeae L.), fue inicialmente mencionada por Morris (1997) y Morris et al. (1998 a b). Posteriormente estos trabajos fueron continuados por Redolfi (1999), Morris et al. (2000), Redolfi et al. (2003, 2004). El presente trabajo complementa los dos últimos mencionados $\mathrm{y}$ tiene como objetivos determinar la distribución espacial y la permanencia temporal de los hormigueros en tres olivares con diferente manejo agronómico, con la finalidad de poder apreciar la respuesta de la comunidad de hormigas a la perturbación producida por el laboreo en el olivar.

\section{Material y Métodos}

Zona de estudio

La zona de estudio se encuentra a $20 \mathrm{Km}$ al norte de la ciudad de Granada (Andalucía). Las observaciones se realizaron en tres olivares diferenciados fundamentalmente por las prácticas agronómicas: un olivar ecológico (Arenales), uno con aplicación de plaguicidas (Colomera 1) y un olivar abandonado durante 10 años y con inicio de laboreo en Julio de 1997 (Colomera 2).

Datos complementarios de la zona de estudio, variedad de olivo y de las variables abióticas pueden ser consultados en Redolfi et al. (1999, 2003, 2004).

Distribución espacial y permanencia temporal de los hormigueros

La distribución espacial intraespecífica y la permanencia temporal de los hormigueros se realizó considerando sólo las 11 especies de Formicidae más abundantes de las 27 registradas en el olivar de Granada (Morris, 1997; Redolfi, 1999). Las hormigas estudiadas fueron: Aphaenogaster gibbosa (Latreille, 1798); A. senilis Mayr, 1853; Messor barbarus (Linneo, 1767); Crematogaster auberti Emery, 1869; C. sordidula (Nylander, 1849); Solenopsis latro Forel, 1894; Tetramorium semilaeve Andrè, 1883; Tapinoma nigerrima (Nylander, 1856); Plagiolepis pygmaea (Latreille, 1798); Camponotus sylvaticus (Olivier, 1792) y Cataglyphis rosenhaueri Santschi, 1925. Para la distribución espacial del total de los nidos se consideraron las 20 especies de hormigas con nidos en el suelo mencionadas por Redolfi et al. (1999, 2004), ya que el resto tienen sus nidos en los árboles, lo que da lugar a otras pautas en la distribución.

En cada olivar se observó el área correspondiente a 60 árboles (seis hileras contiguas de 10 árboles) y los cinco transectos de $100 \mathrm{~m}$ de longitud por un metro de ancho en las calles, entre las hileras de árboles. En el suelo bajo la copa de cada árbol y en las calles se determinó la ubicación de los hormigueros en actividad, en un área total de $540 \mathrm{~m}^{2}$ (9 m²/árbol) y $500 \mathrm{~m}^{2}\left(100 \mathrm{~m}^{2} /\right.$ calle $)$ respectivamente. La observación tuvo una duración de 15 minutos por árbol y por transecto entre las 9:00 y 14:00 horas
Distribución espacial: Se procedió al mapeo de los nidos en actividad y a medir la distancia entre nidos en los meses de Mayo, Julio y Septiembre de 1997.

Permanencia temporal: Se utilizó el mapeo realizado para la distribución espacial y se agregó las observaciones en el mes de Mayo de 1998 para determinar la Permanencia interanual de los nidos.

Análisis de los datos

La distribución espacial de los hormigueros se realizó por medio del método del vecino más próximo (Clark \& Evans, 1954) y la corrección de Donnelly propuesta por Sinclair (1979, en Sinclair, 1985):

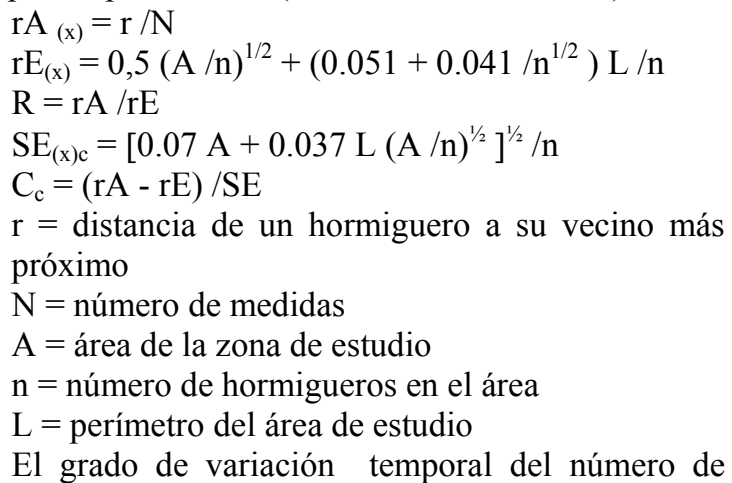
hormigueros fue determinado por medio de los índices de permanencia y de renovación (Cerdá, 1989; Fernández-Escudero, 1994):

-Indice de permanencia (IP): representa el porcentaje de hormigueros que se mantienen respecto al muestreo anterior : $\mathrm{IP}_{\mathrm{i}}=\mathrm{NP}_{\mathrm{i}} / \mathrm{NT}_{\mathrm{i}-1} \times 100$

$\mathrm{NP}_{\mathrm{i}}=$ número de hormigueros que permanecen del muestreo i-1 en el muestreo i.

$\mathrm{NT}_{\mathrm{i}-1}=$ número total de nidos censados en el muestreo anterior.

$\mathrm{IP}_{\mathrm{i}}=$ porcentaje de nidos que permanecen en el muestreo i.

-Indice de renovación (IR): representa el porcentaje de hormigueros nuevos que aparecen en un muestreo : $\mathrm{IR}_{\mathrm{i}}=\mathrm{NN}_{\mathrm{i}} / \mathrm{NT}_{\mathrm{ix}} 100$

$\mathrm{NN}_{\mathrm{i}}=$ número de hormigueros que aparecen en el muestreo i y no aparecieron en el anterior (i - 1)

$\mathrm{NT}_{\mathrm{i}}=$ número de hormigueros censados en el muestreo $\mathrm{i}$

$\mathrm{IR}_{\mathrm{i}}=$ porcentaje de renovación de los hormigueros en el muestreo

Se consideró la permanencia de hormigueros cuando se observó actividad en ellos. Los hormigueros inactivos (comprobados mediante perturbación) se consideraron como ausentes.

\section{Resultados y Discusión}

El mayor número de nidos se presentó en el olivar Colomera 2 (abandonado), seguido por Arenales y Colomera 1, éste último con un escaso número de nidos debido probablemente a la aplicación de plaguicidas (Redolfi, 1999; Redolfi et al., 2004) (Tablas 1 a 3). La mayoría de los nidos estaban ubicados en el área bajo la copa del árbol y sólo $M$. 
barbarus en el olivar abandonado presentó numerosos nidos en las calles debido a la ausencia de laboreo en esta zona (Tabla 3).

Tabla 1. Valores del análisis de la distribución intraespecífica de los hormigueros por el método del vecino más próximo en Arenales, 1997.

\begin{tabular}{llllll}
\hline Mes & Especie & $\mathrm{n}$ & $\mathrm{rA}_{(\mathrm{x})}( \pm \mathrm{D} . \mathrm{S})$. & $\mathrm{R}$ & $\mathrm{P}$ \\
\hline \multirow{2}{*}{ Mayo } & & & & & \\
& & & & & \\
& A. senilis & 16 & $15.03 \pm 8.6$ & 1.63 & $<0.0001$ \\
& T. nigerrima & 8 & $17.28 \pm 9.9$ & 1.27 & $=0.2$ \\
Pulio & P. pygmaea & 24 & $9.42 \pm 8.7$ & 1.27 & 0.02 \\
& & & & & \\
& A. senilis & 23 & $13.50 \pm 9.0$ & 1.79 & 0.0001 \\
& T. nigerrima & 8 & $15.10 \pm 7.8$ & 1.11 & $=0.5$ \\
Septiembre & P. pygmaea & 20 & $13.50 \pm 8.4$ & 1.66 & $<0.0001$ \\
& A. senilis & 5 & $23.75 \pm 11.6$ & 1.31 & $=2$ \\
& C. sordidula & 7 & $19.90 \pm 9.2$ & 1.35 & $=0.1$ \\
& S. latro & 10 & $13.20 \pm 5.3$ & 1.10 & $=0.6$ \\
& T. semilaeve & 6 & $20.20 \pm 8.9$ & 1.25 & $=0.3$ \\
& & & & & \\
\hline
\end{tabular}

Tabla 2. Valores del análisis de la distribución intraespecífica de los hormigueros por el método del vecino más próximo en los olivares Colomera 1 y Colomera 2, 1997.

\begin{tabular}{|c|c|c|c|c|c|c|}
\hline Zona & Mes & Especie & $\mathrm{n}$ & $\mathrm{rA}_{(\mathrm{x})}( \pm \mathrm{D} . \mathrm{S})$. & $\mathrm{R}$ & $\mathrm{P}$ \\
\hline \multirow[t]{3}{*}{ Colomera 1} & Mayo & T. nigerrima & 5 & $20.00 \pm 8.6$ & 1.10 & $=0.7$ \\
\hline & & P. pygmaea & 5 & $20.25 \pm 8.2$ & 1.12 & $=0.6$ \\
\hline & Julio & P. pygmaea & 7 & $22.75 \pm 8.5$ & 1.55 & $=0.002$ \\
\hline \multirow[t]{7}{*}{ Colomera 2} & Mayo & C. auberti & 14 & $15.60 \pm 7.6$ & 1.58 & $=0.0006$ \\
\hline & & T. nigerrima & 53 & $9.30 \pm 3.3$ & 1.90 & $<0.00001$ \\
\hline & & C. rosenhaueri & 5 & $22.10 \pm 17.8$ & 1.39 & $=0.18$ \\
\hline & Julio & C. auberti & 10 & $18.30 \pm 7.1$ & 1.53 & $=0.008$ \\
\hline & & T. nigerrima & 15 & $11.30 \pm 7.9$ & 1.19 & $=0.2$ \\
\hline & Sept. & S. latro & 12 & $16.80 \pm 9.7$ & 1.56 & $=0.002$ \\
\hline & & T. semilaeve & 58 & $8.30 \pm 3.2$ & 1.78 & $<0.0001$ \\
\hline
\end{tabular}

Tabla 3. Valores del análisis de la distribución de los hormigueros de $M$. barbarus por el método del vecino más próximo en los transectos $(\mathrm{n}=5)$. Colomera 2, 1997.

\begin{tabular}{lllll}
\hline Transecto & $\mathrm{n}$ & $\mathrm{rA}_{(\mathrm{x})}( \pm \mathrm{D} . \mathrm{S})$. & $\mathrm{R}$ & $\mathrm{P}$ \\
\hline 1 & 10 & $9.80 \pm 6.3$ & 4.73 & $<0.00001$ \\
2 & 13 & $6.90 \pm 6.9$ & 4.04 & $<0.00001$ \\
3 & 8 & $12.40 \pm 8.9$ & 5.02 & $<0.00001$ \\
4 & 4 & $5.00 \pm 2.6$ & 1.09 & $=0.7$ \\
5 & 11 & $8.25 \pm 1.8$ & 4.77 & $<0.00001$ \\
\hline
\end{tabular}

La ubicación de los hormigueros bajo la copa del árbol fue de preferencia en posición Norte y Este para la mayoría de las especies. Las hormigas de menor tamaño ( $P$. pygmaea, $S$. latro y $T$. semilaeve) construyeron sus nidos bajo piedra y a una distancia no mayor de $50 \mathrm{~cm}$ de la base del tronco, mientras que las especies de mayor tamaño, sólo en algunas ocasiones $(n=7)$ utilizaron la presencia de una piedra para cubrir parte de la entrada del nido a una distancia de 1-1.5 m de la base del tronco. T. nigerrima mostró mayor versatilidad y la entrada de sus nidos se presentaron indistintamente entre los pies de tronco hasta $1.5 \mathrm{~m}$ de estos. La especie $A$. senilis presentó una situación preferencial, en el borde del cuadrado bajo la copa, en el límite con la calle en el lugar más soleado debajo de la copa, en posición S-O.

\section{Distribución espacial intraespecífica de los hormigueros}

La distribución intraespecífica de los hormigueros fue regular y al azar, bajo la copa del árbol y en las calles en los tres olivares (Tablas 1-3). Estos resultados concuerdan en parte con los de Bernstein \& Gobbel (1979), quienes mencionan que la distribución regular de los nidos parece ser la más común entre los formícidos. Igualmente, Levings \& Traniello (1981) recogieron información sobre el patrón de distribución intraespecífica en nidos de 136 especies de hormigas. El patrón mayoritario encontrado fue un espaciamiento regular de los nidos. Una revisión posterior realizada por Ryti \& Case (1992) ratificó este patrón mayoritario.

Las especies A. senilis, M. barbarus, $S$. latro, $T$. semilaeve, $T$. nigerrima y $P$. pygmaea, mostraron una distribución intraespecífica regular de los nidos que pasó a al azar cuando la densidad disminuyó. Mientras que C. sordidula y C. rosenhaueri, con un escaso número de nidos presentaron distribución al azar y $C$. auberti con 14 y 10 nidos tuvo una distribución regular en el olivar Colomera 2, con anterioridad al inicio del laboreo en esta zona (Tablas 1-3).

La distribución del total de los nidos en Arenales y Colomera 2 correspondió a una distribución regular. En cambio, en Colomera 1 apareció sólo regular en el mes de Mayo, con un número mayor de nidos $(n=13)$, pero pasó a al azar en Julio y Septiembre con un número menor $(\mathrm{n}=9, \mathrm{n}=3)$ (Tabla 4$)$.

Estos resultados han sido mencionados por varios autores en otras especies (Waloff \& Balckith, 1962; Bernstein \& Gobbel, 1979; Hölldobler \& Lumsden, 1980; Levings \& Traniello, 1981; FernándezEscudero, 1994). La mayoría de estas especies son territoriales, por lo que al aumentar la densidad, la competencia intraespecífica es mayor y la distribución tiende a hacerse regular (Greenslade, 1975; Harrison \& Gentry, 1981; Ryti \& Case, 1984, 1986; Cushman et al., 1988). En el olivar, el paso de una distribución 
Tabla 4: Valores del análisis de la distribución del total de los hormigueros por el método del vecino más próximo en los tres olivares, durante 1997.

\begin{tabular}{llllll}
\hline Zona & Mes & $\mathrm{n}$ & $\mathrm{rA}_{(\mathrm{x})}( \pm \mathrm{D} . \mathrm{S})$. & $\mathrm{R}$ & $\mathrm{P}$ \\
\hline \multirow{2}{*}{ Arenales } & Mayo & 62 & $7.0 \pm 5.9$ & 1.55 & $<0.00001$ \\
& Julio & 64 & $9.8 \pm 8.5$ & 2.15 & $<0.0001$ \\
& Septiembre & 36 & $8.8 \pm 5.3$ & 1.33 & $=0.0002$ \\
Colomera 1 & Mayo & 13 & $16.5 \pm 12.6$ & 160 & $=0.0006$ \\
& Julio & 9 & $17.8 \pm 10.9$ & 1.40 & $=0.06$ \\
& Septiembre & 3 & $41.0 \pm 16.2$ & 1.61 & $=0.09$ \\
& & & & \\
Colomera 2 & Mayo & 86 & $7.2 \pm 3.7$ & 1.88 & $<0.00001$ \\
& Julio & 44 & $9.8 \pm 7.4$ & 1.82 & $<0.00001$ \\
& Septiembre & 153 & $6.0 \pm 2.8$ & 2.12 & $<0.00001$ \\
\hline
\end{tabular}

Tabla 5. Índices de permanencia y de renovación de nidos durante 1997 en el olivar Arenales.

\begin{tabular}{|c|c|c|c|c|c|c|c|c|}
\hline Zona & Especie & Mes & NT & NP & $\mathrm{NN}$ & ND & IP & IR \\
\hline \multirow[t]{24}{*}{ Arenales } & A. gibbosa & Mayo & 2 & & & & & \\
\hline & & Julio & 1 & 0 & 1 & 2 & 0.0 & 100 \\
\hline & & Septiembre & 0 & 0 & 0 & 0 & 0.0 & 0.0 \\
\hline & A. senilis & Mayo & 16 & & & & & \\
\hline & & Julio & 23 & 5 & 18 & 11 & 31.2 & 78.2 \\
\hline & & Septiembre & 5 & 1 & 4 & 20 & 4.3 & 80.0 \\
\hline & C. sordidula & Mayo & 1 & & & & & \\
\hline & & Julio & 0 & 0 & 0 & 0 & 0.0 & 0.0 \\
\hline & & Septiembre & 7 & 0 & 7 & 0 & 0.0 & 100 \\
\hline & T. semilaeve & Mayo & 3 & & & & & \\
\hline & & Julio & 2 & 0 & 2 & 3 & 0.0 & 100 \\
\hline & & Septiembre & 6 & 0 & 6 & 2 & 0.0 & 100 \\
\hline & T. nigerrima & Mayo & 8 & & & & & \\
\hline & & Julio & 8 & 0 & 8 & 8 & 0.0 & 100 \\
\hline & & Septiembre & 3 & 0 & 3 & 8 & 0.0 & 100 \\
\hline & P. pygmaea & Mayo & 24 & & & & & \\
\hline & & Julio & 20 & 2 & 18 & 22 & 8.3 & 90 \\
\hline & & Septiembre & 1 & 0 & 1 & 20 & 0.0 & 100 \\
\hline & C. sylvaticus & Mayo & 2 & & & & & \\
\hline & & Julio & 2 & 0 & 2 & 2 & 0.0 & 100 \\
\hline & & Septiembre & 2 & 0 & 2 & 2 & 0.0 & 100 \\
\hline & $\begin{array}{l}C . \\
\text { rosenhaueri }\end{array}$ & Mayo & 1 & & & & & \\
\hline & & Julio & 2 & 0 & 2 & 2 & 0.0 & 100 \\
\hline & & Septiembre & 2 & 0 & 2 & 2 & 0.0 & 100 \\
\hline
\end{tabular}

$\mathrm{x} \pm$ D.S: $\mathrm{IP}=2.7 \pm 7.9 . \mathrm{IR}=84.3 \pm 33.7$

NT : hormigueros existentes en el muestreo, NP : hormigueros que permanecen del muestreo anterior, $\mathrm{NN}$ : hormigueros nuevos aparecidos respecto al muestreo anterior, ND : hormigueros desaparecidos respecto al muestreo anterior, IP : índice de permanencia, IR : índice de renovación.

de los nidos al azar a una distribución regular al aumentar la densidad, podría deberse también a la competencia intraespecífica. Sin embargo, la simple información espacial no puede ser usada por sí sola, como prueba de interacciones competitivas (Pielou, 1960; Brown \& Orians, 1970; Ryti \& Case, 1992). La afirmación o no de la existencia de territorio, depende más de la demostración de diferentes mecanismos conductuales que de un tipo $\mathrm{u}$ otro de distribución espacial (Hölldobler \& Lumsden, 1980). La confirmación de esta relación, entre un patrón de distribución regular de nidos de una especie y un comportamiento territorial, implica conocer diversos parámetros de su biología (Gómez \& Espadaler, 1996).

Por otro lado, si los lugares para anidar son escasos, la distribución de éstos puede influir en la de los hormigueros (Levings \& Traniello, 1981). En el caso del olivar, con un marco de plantación regular de $10 \times 10 \mathrm{~m}$ entre pie de árbol, un intenso laboreo en las calles y con una vegetación bajo su copa, el árbol es un importante recurso alimenticio y el suelo bajo su copa un lugar apropiado de anidación por las condiciones microclimáticas favorables. El área bajo la copa (540 $\mathrm{m}^{2} / 60$ árboles) es escasa en comparación con el área total (4 $929 \mathrm{~m}^{2} / 60$ árboles) y sin embargo en ella se encontraron los nidos de la mayoría de las especies, mientras que en las calles principalmente los nidos de $M$. barbarus en el olivar abandonado.

\section{Permanencia temporal de los hormigueros}

Intraanual- La ubicación de los nidos de las diferentes especies varió de una manera dinámica y la mayoría de los nidos no permanecieron a lo largo de la estación (Tablas 5 y 6). Los índices de permanencia de los nidos fueron muy bajos (Arenales: 2.7\%, Colomera 1: $10.0 \%$ y Colomera 2: $11.2 \%$ ), mientras que los índices de renovación fueron generalmente altos $(78.2 \%$ a $100 \%)$, lo que se observó mejor en los olivares con un mayor número de especies (Arenales y Colomera 2), lo cual indica que ha existido complementariedad de los índices de permanencia y renovación, y los hormigueros que desaparecían eran sustituidos por otros de nueva creación. Esta dinámica podría ser sumamente importante para el sistema considerando las ventajas que representan para el suelo los hormigueros, como son la aireación por el movimiento del suelo e incorporación de materia orgánica. En el olivar de Arenales, P. pygmaea con 24 nidos en el mes de Mayo, disminuyó a 20 en el mes de Julio, pero éstos representaron sólo el $8.0 \%$ de los nidos del mes de Mayo, desapareciendo estos nidos en el mes de Septiembre. Igualmente, $A$. senilis no mostró permanencia de sus nidos y $T$. nigerrima presentó $31.5 \%-12.5 \%$ (Tabla 5). C. auberti, con el mayor índice de permanencia (57.1\%) entre Mayo y Julio en Colomera 2, tuvo un bajo índice de 
renovación $(20 \%)$ en ese mes y $0.0 \%$ para ambos índices entre Julio y Septiembre, debido al laboreo en la zona a partir del mes de Julio. Igualmente, $T$. nigerrima, sólo mantuvo el $10.9 \%$ de los nidos entre concluido el laboreo en este olivar, se comprobó que aparecen de nuevo nidos de esta especie, pero en las calles. En ocasiones, se pudo observar directamente formación de nuevos hormigueros en las calles por fisión de nidos situados bajo la copa. Estos resultados indican una dinámica muy intensa a causa del disturbio producido por las diferentes faenas del laboreo en el cultivo. Mayo y Julio. En el mes de Septiembre, una vez

\section{Agradecimientos}

El presente trabajo se ha realizado con el financiamiento de la Junta de Andalucía y la Beca de la AECI (Mutis) a I. Redolfi.

\section{Literatura citada}

Adlung K.G. 1966. A critical evaluation of the european research on use of red wood ants (Formica rufa group) for the protection of forests against harmful insects. Dpto. Agricultural Research Service, París. 57: 167-189.

Andersen A.N. 1990. The use of ant communities to evaluate change: in Australian terrestrial ecosystem review and a recipe. Proc. Ecol. Soc. Aust. 16: 3-57.

Bernstein R.A. \& Gobbel M. 1979. Partitioning of space in communities of ants. J. Anim. Ecol. 48: 931942.

Tabla 6. Índices de permanencia y de renovación de nidos durante 199' Brown J.L. \& Orians G.H. 1970. Spacing en Colomera 1 y Colomera 2.

\begin{tabular}{lllllllll}
\hline Zona & Especie & Mes & NT & NP & NN & ND & IP & IR \\
\hline \multirow{2}{*}{ Colomera 1 } & T nigerrima & Mayo & 5 & & & & & \\
& & Julio & 2 & 1 & 1 & 4 & 20.0 & 50.0 \\
& & Septiembre & 3 & 0 & 3 & 2 & 0.0 & 100 \\
& P. pygmaea & Mayo & 5 & & & & & \\
& & Julio & 7 & 1 & 6 & 4 & 20.0 & 85.7 \\
& Septiembre & 0 & 0 & 0 & 7 & 0.0 & 0.0 \\
\hline
\end{tabular}

$x \pm$ D.S.: $I P=10.0 \pm 11.5, I R=58.9 \pm 44.6$

\begin{tabular}{|c|c|c|c|c|c|c|c|c|}
\hline \multirow[t]{6}{*}{ Colomera 2} & \multirow[t]{3}{*}{ C. auberti } & Mayo & 14 & & & & & \\
\hline & & Julio & 10 & 8 & 2 & 6 & 57.1 & 20 \\
\hline & & Septiembre & 0 & 0 & 0 & 0 & 0.0 & 0.0 \\
\hline & \multirow[t]{3}{*}{ T. nigerrima } & Mayo & 46 & & & & & \\
\hline & & Julio & 9 & 5 & 4 & 41 & 10.9 & 44.4 \\
\hline & & Septiembre & 1 & 0 & 1 & 1 & 0.0 & 100 \\
\hline \multirow[t]{9}{*}{ Calles: } & \multirow[t]{3}{*}{ M. barbarus } & Mayo & 12 & & & & & \\
\hline & & Julio & 10 & 0 & 10 & 12 & 0.0 & 100 \\
\hline & & Septiembre & 46 & 3 & 43 & 7 & 30.0 & 93.5 \\
\hline & \multirow[t]{3}{*}{ T. nigerrima } & Mayo & 7 & & & & & \\
\hline & & Julio & 7 & 1 & 6 & 6 & 14.2 & 85.7 \\
\hline & & Septiembre & 12 & 0 & 12 & 7 & 0.0 & 100 \\
\hline & \multirow[t]{3}{*}{ C. rosenhauer } & Mayo & 5 & & & & & \\
\hline & & Julio & 1 & 0 & 1 & 5 & 0.0 & 100 \\
\hline & & Septiembre & 2 & 0 & 2 & 1 & 0.0 & 100 \\
\hline
\end{tabular}

$\mathrm{x} \pm$ D.S.: $\mathrm{IP}=11.2 \pm 18.9, \mathrm{IR}=74.4 \pm 38.2$

NT : hormigueros existentes en el muestreo, NP : hormigueros que permanecen del muestreo anterior, $\mathrm{NN}$ : hormigueros nuevos aparecidos respecto al muestreo anterior, ND : hormigueros desaparecidos respecto al muestreo anterior, IP : índice de permanencia, IR : índice de renovación.

Interanual- El índice de permanencia de los hormigueros de todas las especies, tanto bajo los árboles como en las calles, entre 1997 y 1998 fue cero, lo cual era de esperar en este agroecosistema, muy diferente a lo que ocurre en un habitat natural, en donde los nidos pueden tener un IP interanual, tal como menciona Fernández-Escudero (1994) para Proformica longiseta Colligwood, con un IP del 60\% en el Valle del Río San Juan en el macizo de Sierra Nevada. patterns in mobile animals. Ann. Rev. Ecol. Syst. 1: 239-262.

Burbidge A.H., Leicester K., McDavitt S. \& Majer J.D. 1992. Ants as indicators of disturbance at Yanchep National Park, Western Australia. J. Roy. Soc. West. Aus. 75: 89-95. Cerdá X. 1989. La policladia y el transporte mutuo en la hormiga Cataglyphis iberica (Emery, 1906) Tesis doctoral. Universidad Autónoma de Barcelona. España.

Cushman J.H., Martinsen G.D. \& Mazeroli A.I. 1988. Density and size dependent spacing of ant nests: evidence for intraspecific competition. Oecología. 77: 522-525.

Clark P.J. \& Evans F. 1954. Distance to nearest neighbour as a measure of spatial relantionships in populations. Ecology. 35(4): 445-453.

Detrain C. 1990. Field study on foraging by the polymorphic ant species, Pheidole pallidula. Ins. Soc. 37(4): 315-332.

El Haidari H.S. 1981. The use of predator ants for the control of date palm insect pests in the Yemen Arab republic. Date Palm J. 1(1): 129132.

Fernández-Escudero J. 1994. Estudio de la biología de Proformica longiseta Collingwood, 1978 (Hymenoptera: Formicidae). Tesis Doctoral. Universidad de Granada. España.

Fiala B., Grunsky H., Maschwitz U. \& Linsenmair K.E. 1994. Diversity of ant-plant interactions: protective efficacy in Macaranga species with different degrees of ant association. Oecología. 97: 186-192.

Gomez C. \& Espadaler X. 1996. Distancias de forrageo, áreas de forrageo y distribución espacial de nidos de Aphaenogaster senilis Mayr (Hym. Formicidae). Miscelánia Zoológica. 19 (2): 19-25.

Greenslade P.J.M. 1975. Dispersion and history of a population of the meat ant Iridomyrmex purpureus.

Harrison J.S. \& Gentry J.B. 1981. Foraging pattern, colony distribution and foraging range of the Florida harvester ant Pogonomyrmex badius. Ecology. 62(6): 1467-1473.

Hölldobler B. \& Lumsden C.J. 1980. Territorial strategies in ants. Science. 210: 732-739. Aust. J. Zool. 23: 495-510. 
Levings S.C. \& Traniello F.A. 1981. Territoriality, nest dispersion and community structure in ants. Psyche. 88: 265-319.

Majer J.D. 1972. The ant mosaic in Ghana cocoa farms. Bull. Ent. Res. 62: 151-160.

1983. Ants: Bio-Indicators of Minesite Rehabilitation, Land-Use, and Land Conservation. Env. Manag. 7(4): 375-383.

. 1994. Introduction of ants as potential biological control agents, with particular reference to cocoa. Harvest. 16(1, 2): 1-4.

Majer J.D. \& Delabie J.H.C. 1993. An evaluation of Brasilian cocoa farm ants as potential biological control agents. Journal of Plant Protection in the Tropics. 10(1): 43-49.

1994. Comparison of the ant communities of annually inundated and terrafirme forests a Trombetas in the Brazilian Amazon. Ins. Soc. 4: 343-359.

Majer J.D. \& Beeston G. 1996. The Biodiversity Integrity Index: An Ilustration Using ants in Western Australia. Cons. Biol. 10(1): 65-73.

Morris T.I. 1997. Interrelaciones entre olivos, plagas y depredadores. Tesis Doctoral. Universidad de Granada. España.

Morris T.I., Campos M., Jervis M.A., McEwen P.K. \& Kidd N.A.C. 1998a. Potential effects of various ant species on green lacewing, Chrysoperla carnea (Stephens) (Neuropt., Chrysopidae) egg numbers. J. Appl. Ent. 122: 401-403.

Morris T.I., Symondson W.O.C., Kidd N.A.C., Jervis M.A. \& Campos M. 1998b. Are ants significant predator of the olive moth, Prays oleae?. Crop Protection (en prensa).

Morris T.I., Symondson W.O.C., Kidd N.A.C. \& Campos M. 2002. The effect of different ant species on the olive moth, Prays oleae (Bern.), in Spanish olive orchard. J. Appl. Ent. 126: 1-6.

Paulson G.S. \& Akre R.D. 1992. Evaluating the effectiveness of ants as biological control agents of pear Psylla (Homopera: Psyllidae). J. Econ. Ent. 81(1): 7073.

Peng R.K., Christian K. \& Gibb K. 1997. Distribution of the green ant, Oecophylla samaragdina (F.) (Hymenoptera: Formicidae), in relation to native vegetation and the insect pests in cashew plantations in Australia. International Journal of Pest Management. 43(3): 203211.

Perfecto I. \& Snelling R. 1995. Biodiversity and the transformation of a tropical agroecosystem: ants in coffee plantations. Ecol. Appl. 5(4): 1084-1097.

Perfecto I. \& Vandermeer J. 1994. Understanding biodiversity loss in agroecosystems: Reduction of ant diversity resulting from transformation of the coffee ecosystem in Costa Rica. Entomol. 2: 7-13.
Petal J. 1978. The role of the ants in ecosystems. : 245-292. In: Production ecology of ants and termites. Brian M.V. International Biological Programme, $N^{\circ}$ 13. Cambridge University Press. Cambridge.

Pielou E.C. 1960. A single mechanism to account for regular, random and aggregated populations. J. Ecol. 48: 575-584.

Rabitsch W.B. 1997. Tissue-specific accumulation patterns of $\mathrm{Pb}, \mathrm{Cd}, \mathrm{Cu}, \mathrm{Zn}, \mathrm{Fe}$, and $\mathrm{Mn}$ in workers of three ant species (Formicidae, Hymenoptera) from a metalpolluted site. Arch. Environ. Contam. Toxicol. 32: 172177.

Redolfi I. 1999. Las hormigas (Hymenoptera: Formicidae) en el agroecosistema del olivo en Granada, España. Tesis Doctoral. Universidad de Granada. España.

Redolfi I., Tinaut A., Pascual F. \& Campos M. 1999. Qualitative aspects of myrmecocenosis (Hym., Formicidae) in olive orchards with different agricultural management in Spain. J. Appl. Ent. 123: 621-627.

2003. Patrón de actividad de Tapinoma nigerrima (Nylander) y Crematogaster scutellaris (Olivier) (Hymenoptera, Formicidae) en el cultivo de olivo y en el laboratorio. Zool. baetica. 13/14: $37-55$.

2004. Densidad de nidos de la comunidad de hormigas (Formicidae) en tres olivares con diferente manejo agronómico en Granada, España. Ecol. apl. 3(1 y 2): 73-81.

Ryti R.T. \& Case T.J. 1984. Spatial arrangement and diet overlap between colonies of desert ants. Oecología. 62: 401-404.

1986. Overdispersion of ant colonies: a test hypothesis. Oecologia. 69: 446-453.

1992. The role of neighborhood competition in the spacing and diversity of ant communities. Am. Nat. 139: 55-74.

Sinclair D.F. 1985. On tests of spatial randomness using mean nearest neighbor distance. Ecology. 66 (3): 10841085.

Tinaut A., Jiménez-Rojas J. \& Pascual R. 1994. Estudio de la mirmecofauna de los bosques de Quercus Linneo, 1753, de la provincia de Granada (Hymenoptera : Formicidae). Ecología. 8: 429-438.

Waloff N. \& Balckith R.E. 1962. The growth and distribution of the mounds of Lasius flavus (Fabricius) in silwood park, Belkshire. J. Anim. Ecol. 31: 421-437.

Way M.J. \& Khoo K.C. 1992. Role of ant in pest management. Ann. Rev. Entomol. 37: 479-503.

Weseloh R.M. 1993. Manipulation of Forest Ant (Hymenoptera: Formicidae) abundance and resulting impact on Gypsy Moth (Lepidoptera: Lymantriidae) populations. Env. Ent. 22(3): 587-594.

1996. Effect of supplemental foods on foraging behavior of forest ants in Connecticut. Env. Ent. 25(4): 848-853.

\footnotetext{
${ }^{1}$ Estación Experimental del Zaidín, Profesor Albareda 1, Granada, España (1996-1999). Universidad Nacional Agraria La Molina, Av. La Molina s/n, La Molina, Lima, Perú. rhi@lamolina.edu.pe

2 Estación Experimental del Zaidín (CSIC) Granada, España. fruano@ugr.es, mcampos@eez.csic.es.

${ }^{3}$ Universidad de Granada, Campus Universitario Fuentenueva. 18071. Granada. España. hormiga@ugr.es, fpascual@ugr.es.
} 